Documentation et bibliothèques

DOCUMENTATION BIBLIOTHËQUES

\title{
Les bibliothèques centrales de prêt du Québec et de la
} Colombie Britannique Organisation et modes de fonctionnement

\section{Jacques Panneton et Philippe Sauvageau}

Volume 19, numéro 1, mars 1973

URI : https://id.erudit.org/iderudit/1050958ar

DOI : https://doi.org/10.7202/1050958ar

Aller au sommaire du numéro

\section{Éditeur(s)}

Association pour l'avancement des sciences et des techniques de la documentation (ASTED)

\section{ISSN}

0315-2340 (imprimé)

2291-8949 (numérique)

Découvrir la revue

\section{Citer cet article}

Panneton, J. \& Sauvageau, P. (1973). Les bibliothèques centrales de prêt du Québec et de la Colombie Britannique : organisation et modes de fonctionnement. Documentation et bibliothèques, 19(1), 8-19.

https://doi.org/10.7202/1050958ar

\section{Résumé de l'article}

Les auteurs font une analyse comparative des bibliothèques centrales de prêt existant actuellement au Québec et en Colombie Britannique. Ils en exposent la structure, le mode de fonctionnement et de financement, les services offerts, et terminent par de brefs commentaires concernant l'automatisation des diverses opérations.
Tous droits réservés (C) Association pour l'avancement des sciences et des techniques de la documentation (ASTED), 1973
Ce document est protégé par la loi sur le droit d'auteur. L'utilisation des services d'Érudit (y compris la reproduction) est assujettie à sa politique d'utilisation que vous pouvez consulter en ligne.

https://apropos.erudit.org/fr/usagers/politique-dutilisation/ 
Les bibliothèques centrales de prêt du Québec et de la Colombie Britannique

\section{ORGANISATION ET MODES DE FONCTIONNEMENT}

par Jacques Panneton et Philippe Sauvageau administrateurs délégués:

Bibliothèque centrale de prêt de la Mauricie;

Bibliothèque centrale de prêt de l'Outaouais

et du

Saguenay-Lac-Saint-Jean.

Les auteurs font une analyse comparative des bibliothèques centrales de prêt existant actuellement au Québec et en Colombie Britannique. Ils en exposent la structure, le mode de fonctionnement et de financement, les services offerts, et terminent par de brefs commentaires concernant l'automatisation des diverses opérations.

\section{AVERTISSEMENT}

Le Québec de 1973 compte trois bibliothèques centrales de prêt, en activité dans la Mauricie, dans l'Outaouais et au SaguenayLac-Saint-Jean. Leur vocation première est de pourvoir en lecture publique les régions rurales qu'on leur a désignées. Cette communication veut décrire brièvement leur organisation et leurs modes de fonctionnement dans ce qu'ils ont de commun, et les comparer avec celles de la Colombie Britannique.

Chacune a ses caractéristiques propres, malgré de très grandes similitudes, et aucune ne prétend être le remède universel à tous les maux, ni même avoir réglé tous ses problèmes. Pourquoi l'une prête-t-elle des oeuvres d'art et l'autre non? pourquoi la cadette n'utilise-t-elle pas de bibliobus? Pourquoi, en Mauricie, le prêt per capita par l'intermédiaire du bibliobus double-t-il celui des succursales (6.1 contre 3.5)? Autant de questions complexes et passionnantes auxquelles nous tenterons de répondre. Mais il faut bien choisir pour n'avoir pas à tout écrire la première fois. Cherchez donc seulement ce qu'on veut y mettre.

Si la lecture vous laisse l'impression du plus grand confort moral, méfiez-vous: quand on a des cheveux blancs à vingt-neuf ans, on laisse la sérénité aux plus âgés!

\section{La direction des bibliothèques publiques du Québec}

C'est en 1959 seulement que le Québec a adopté une loi des bibliothèques publiques. Elle créait deux organismes complémentaires qui sont aujourd'hui placés sous la juridiction du Ministère des Affaires culturelles.

La Commission des bibliothèques publiques du Québec est consultative; elle comprend le directeur des bibliothèques publiques et six autres membres nommés par le Conseil des Ministres pour leur intérêt à la cause des bibliothèques. Sa fonction est de formuler au ministre ses constatations et opinions et de lui recommander les moyens qu'elle juge les meilleurs pour établir et développer un réseau de bibliothèques publiques.

Le Service des bibliothèques publiques lui est administratif; il est constitué du directeur des bibliothèques publiques et des fonctionnaires jugés nécessaires. De manière générale, il doit veiller à l'exécution de la loi, orienter la Commission par ses recherches, diriger les bibliothèques publiques vers la meilleure efficacité, appliquer les directives du ministre et la politique de la Commission, administrer le budget que lui accorde le gouvernement.

Le premier geste de cette direction fut, en 1960 , la réalisation d'une enquête auprés des municipalités et bibliothèques publiques du Québec. On l'aurait deviné, le Québec était bon dernier au Canada dans tous les domaines: nombre de bibliothèques, de bibliothécaires, de volumes, de prêts per capita, montant des revenus, des contributions municipales, des subventions provinciales. En vue d'une action solide, la direction des bibliothèques publiques a préparé une réglementation détaillée concernant les subventions du gouvernement aux bibliothèques publiques. Quelle que soit l'opinion qu'on en ait, quelle que soit l'opinion de la Commission ellemême qui n'arrive plus à respecter ses propres règlements à cause des faibles budgets dont elle bénéficie (?), il faut bien admettre que, de 1960 à 1971,

a) les contributions municipales sont passées de $\$ 1,093,000$ à $\$ 4,730,000$;

b) les subventions aux bibliothèques publiques sont passées de $\$ 140,000$ à $\$ 1$,557,000 ;

c) le nombre des bibliothèques municipales est passé de 21 à 73;

d) le nombre des bibliothèques subventionnées est passé de 71 à 114;

e) le nombre d'usagers est passé de 331,000 à 814,000 ;

f) le nombre de volumes est passé de 1,830,000 à 4,265,000 (1.22 per capita);

g) le nombre de volumes prêtés est passé de $3,928,000$ à $10,305,000$ (2.96 per capita);

h) les recettes totales sont passées de $\$ 1$,554,000 à $\$ 7,333,000(\$ 2.10)$.

Toutefois, ces chiffres ne devraient pas nous empêcher de réfléchir, peut-être même à haute voix, au chemin qu'il reste à parcourir: il faut bien courir quand on n'est pas parti à temps!

Au Québec comme ailleurs, lorsqu'on a décidé de pourvoir en bibliothèques publiques les régions rurales, la seule solution que l'on ait trouvée fut la bibliothèque régionale que I'on a baptisée "bibliothèque centrale de prêt." Après 10 ans, le Québec en compte trois (déjà ou seulement?) desservant une population totale de 236,600 âmes, dont 73,000 usagers qui ont emprunté 831,173 volumes en 1971-72. 


\section{1-A ${ }^{1}$ La structure des bibliothèques publiques de la Colombie Britannique.}

La population de la Colombie Britannique a bénéficié très tôt d'un réseau structuré de bibliothèques publiques, réseau conçu à partir de deux besoins fondamentaux de l'homme: l'information et la récréation. Cette dernière fonction de la bibliothèque publique, importante en raison de l'augmentation des heures de loisir, n'éclipse pourtant pas celle de l'information. Les changements rapides, les modifications des structures, les problèmes complexes causés par l'explosion démographique présupposent, pour l'individu, l'accès rapide et gratuit à l'information et à la documentation, afin de l'aider à prendre des décisions éclairées face à de multiples situations.

II est utopique de croire que "l'éducation permanente" existe par l'école seulement. La télévision, les moyens de communication, les bibliothèques publiques en tant que partie d'une structure de diffusion culturelle sont autant d'éléments essentiels qui forment ce que l'on appelle de plus en plus l'éducation permanente. Cette fonction n'est pas seulement le fait d'un adulte qui retourne à l'école pour suivre un cours de "cuisine"... II y aura toujours des milliers d'individus qui ne retourneront plus à l'école et d'autres qui y retourneront pour une ou deux sessions seulement. Cette forme d'apprentissage "maître-élève" ne peut remplacer les structures tels les centres culturels et les bibliothèques publiques qui se situent au centre du concept "loisir".

Les Colombiens ont compris l'importance des bibliothèques publiques. En 1970, les bibliothèques publiques de la Colombie desservaient 1,714,138 personnes sur une population globale de $2,139,000$; elles prêtaient $14,359,161$ volumes ou près de 7 volumes par tête et dépensaient $\$ 6,431,645$ ou $\$ 3.70$ par tête.

Le réseau des bibliothèques publiques de la Colombie est composé de deux éléments bien distincts: les bibliothèques municipales et les bibliothèques régionales ou centrales de prêt. Elles sont coiffées par la Commission pour le développment des bibliothèques.

\section{La Commission pour le développement des bibliothèques} A. Composition

La Commission est formée de cinq membres nommés par le lieutenant-gouverneur en conseil.

\section{B. Objectif majeur}

La commission pour le développement des bibliothèques est un organisme gouvernemental qui encourage et stimule l'établissement et le développement des bibliothèques publiques.

\section{Moyens d'action}

1. Le " $A$ " venant après certains numéros indique que le texte qui suit est l'exposé de ce qui existe, à ce chapitre, en Colombie Britannique.
- La commission détient les pouvoirs d'appliquer la loi régissant les bibliothèques publiques, verse les subventions aux bibliothèques publiques (en 1970, elle distribua $\$ 392,522$.), prépare des projets de planification, aide les bibliothèques publiques sur le plan professionnel, compile les statistiques annuelles, établit des normes qualitatives, publie certains documents spécialisés, joue un rôle d'agent de liaison auprès des autres organismes gouvernementaux;

- De son siège social à Victoria et des succursales de Prince George, Dawson Creek et Cranbrook, elle envoie par la poste, frais de port payé, les volumes qui lui sont demandés par des lecteurs vivant là où il n'y a aucun service de bibliothèques publiques. Ce service existe depuis 1919;

- La Commission dirige trois succursales, dont l'une est dotée d'un bibliobus. Le personnel professionnel y est engagé et payé par la Commission. Ce service prend fin lorsque les municipalités de la région décident de créer une bibliothèque régionale:

- Toute demande venant de 10 personnes et plus est prise en considération par la Commission. Elle dépose de 150 à 200 volumes dans les communautés encore privées de service de bibliothèques publiques et ces volumes sont échangés tous les quatre mois;

- La commission s'occupe aussi de prêt inter-bibliothèques à partir de son siège social à Victoria. En 1970, ce service recevait 2,872 demandes;

- La Commission prône actuellement une coopération intensive entre les bibliothèques. Un Comité fut mis sur pied par la Commission afin de formuler des recommandations relatives à cette question.

\section{Les trois bibliothèques centrales de prêt du Québec Notes historiques}

La Bibliotheqque centrale de prêt de la Mauricie a été fondée en 1961, fruit d'une convergence de préoccupations entre le "vouloir lire" régional d'une part, groupant principalement la Bibliothèque de Trois-Rivières, une demi-douzaine d'administrations municipales, la Société Saint-Jean-Baptiste et nombre d'individus, et, d'autre part, la Commission des bibliothèques publiques du Québec, mise au défi par l'enquête de 1960. D'abord jumelée avec la Bibliothèque de Trois-Rivières, elle s'en détache rapidement et devient très vite la plus imporante bibliothèque publique de la région. En 1971-72, elle dessert 91,000 âmes dont 26,800 abonnés auxquels elle préte 345,451 volumes, soit 4.2 volumes per capita, avec une collection de 118,328 volumes. En plus des livres, elle compte une collection de 800 films et une discothèque de 6,000 disques. Son territoire englobe maintenant les régions 10 et 15 telles que définies dans le Découpage du Territoire Québécois en régions de bibliothèques. Le siège social est à TroisRivières. 
La Bibliothèque centrale de prêt de l'Outaouais, pour sa part, naît en 1964 à la suite des efforts d'un Comité provisoire qui fit effectuer une enquête par monsieur Claude Aubry, directeur de la Bibliothèque municipale d'Ottawa, en 1962. L'expérience de la Mauricie étant concluante, la Commission des bibliothèques publiques du Québec accepte rapidement le projet. Logée à l'enseigne de la bibliothèque municipale de Hull, comme la $\mathrm{Bi}$ bliothèque centrale de prêt de la Mauricie à Trois-Rivières, elle s'en détache plus rapidement encore. En 1971-72, elle dessert 117,800 âmes dont 40,528 abonnés auxquels elle a prêté 407,472 volumes, soit 3.4 volumes per capita, avec une collection de 142,529 volumes. Elle compte également une cinémathèque de 1,176 films, une discothèque de 4,300 disques, une collection de 491 oeuvres d'art. Son territoire comprend les régions de bibliothèques 19 et 20 . Son siège social est à Hull.

La Bibliothèque centrale de prêt du Saguenay-Lac-Saint-Jean est la cadette, l'année 1971-72 étant celle de sa fondation. Dès le départ, elle s'établit dans son immeuble à Alma. Son territoire englobe les régions de bibliothèques 4 et 5 . Après une année d'activité seulement, elle atteint 29,000 âmes dont 5,700 abonnés et prête 103,197 volumes. Le prêt per capita sera plus significatif en 1972-73. Ses collections comprennent 36,896 livres et 1,769 disques.

\section{2-A. Bibliothèques régionales ou bibliothèques centrales}

La Bibliothèque régionale de l'lle de Vancouver fondée en 1930, a prêté, en $1971,1,478,228$ volumes aux 145,000 adultes et enfants inclus dans son territoire, avec une collection de 240,000 volumes. Cette bibliothèque dessert les municipalités de l'lle de Vancouver à l'exception de Victoria qui possède sa propre bibliothèque municipale. Le siège social est situé à Nanaimo. En plus des livres, elle offre aux succursales plus de 500 oeuvres d'art destinées au prêt individuel.

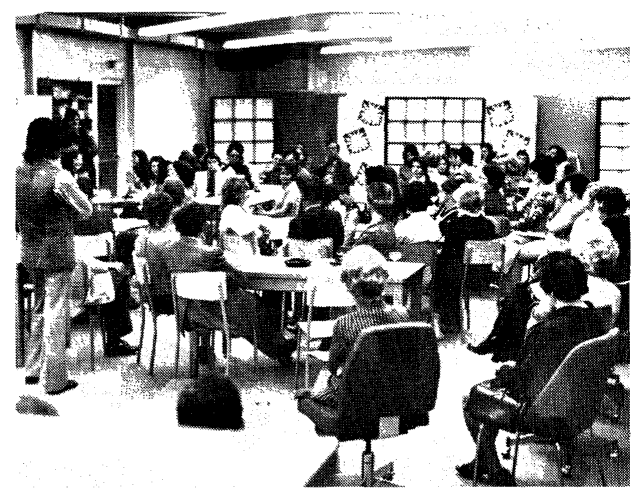

Bibliothèque centrale de prêt de la Mauricie: les responsables de succursales en atelier (au total, 100 présences).
Une partie infime de la clientèle est desservie par le bateau appartenant à l'Eglise Unie. On y trouve de même une collection de volumes imprimés en gros caractères pour les personnes souffrant de déficience visuelle.

La Bibliothèque régionale de Fraser Valley fondée en 1934, possède une collection de 349,810 livres et 1,031 films. La population desservie, 372,301 personnes, a emprunté 1,992,155 volumes et 12,744 films. II y a 83,825 usagers. Le personnel technique a catalogué et préparé matériellement 72,379 volumes. Le siège social, établi à Abbotsford, a reçu et expédié 83,796 demandes spéciales.

La Bibliothèque régionale d'Okanagan fondée en 1936, possède une collection de 140,584 volumes et de 787 films. Elle dessert une population de 98,228 personnes. $\mathrm{La} \mathrm{Bi}$ bliothèque régionale a reçu 19,793 demandes spéciales. Le directeur remarque que le fait de mettre à la disposition des succursales un catalogue complet des volumes de la bibliothèque, grâce à l'ordinateur, a augmenté de $48 \%$ les demandes spéciales. Il y eut l'addition d'un second bibliobus. Les 45,364 usagers ont emprunté 806,113 volumes et 2,667 films.

\section{Statuts légaux}

Les trois Bibliothèques centrales de prêt du Québec sont des corporations privées incorporées en vertu de la troisième partie de la Loi des Compagnies du Québec, c'est-àdire, des compagnies sans but lucratif. Elles sont régies par des règlements généraux identiques, à quelques vétilles près. Les trois paliers d'autorité sont l'assemblée générale, le conseil d'administration et le bureau.

L'assemblée générale est composée de quatre catégories de membres: en catégorie "a", on retrouve les signataires de la requête en constitution de la corporation; en catégorie " $b$ ", le secrétaire de la Commission des Bibliothèques publiques du Québec et les personnes nommées par la Commission, en nombre indéfini; en catégorie "c", l'administrateur délégué, ou le bibliothécaire en chef; et en catégorie " $d$ ", chacun des représentants des municipalités contractantes. La majorité des membres de ces corporations sont donc des représentants des municipalités qui ont convenu de participer à la Bibliothèque centrale de prêt et non ces municipalités elles-mêmes. Par le biais du choix des reprsentants, ces dernières sont néanmoins en mesure de contrôler elles-mêmes l'organisme auquel elles participent. On notera qu'aucun pouvoir scolaire n'a de représentation au sein de la bibliothèque centrale de prêt. L'assemblée générale siège au moins une fois l'an.

Le conseil d'administration élu par l'assemblée générale doit respecter un partage prédéfini de représentation: huit membres dont quatre de catégorie " $b$ ", indirectement de la Commission des bibliothèques publiques qui, historiquement, les a choisis parmi des représentants de municipa- 
lités, l'administrateur délégué et trois autres provenant au choix de la catégorie " $d$ " seulement ou des catégories "d" et "a", mais un seulement de la catégorie "a". On pourrait invoquer de multiples raisons pour justifier ce partage: elles sont techniques pour une part et fiscales pour une autre, notamment le partage inégal du fardeau financier entre les municipalités et le gouvernement provincial. Le conseil d'administration siège au moins quatre fois l'an.

Le bureau, pour sa part, assume des responsabilités administratives spécifiques, mais ni lui ni aucun de ses membres ne détient de pouvoirs autres que ceux que lui aura spécifiquement accordés le conseil d'administration.

L'administrateur délégué a la responsabilité de l'oragnisation efficace, du développement et du rendement maximum de la bibliothèque centrale de prêt. C'est à ce titre qu'il est assuré d'une présence au sein de l'assemblée générale, du conseil d'administration et du bureau. A noter cependant que le règlement général lui réserve tous les pouvoirs sur le plan professionnel, en tant que bibliothécaire.

\section{3-A. Statuts légaux}

Les bibliothèques régionales de la Colombie sont, au sens légal, des compagnies administrées, comme au Québec, par un conseil d'administration formé des représentants dûment désignés par les municipalités affiliées. Chaque municipalité et chaque district scolaire, un tel district ayant juridiction sur les territoires non organisés en municipalités, ont droit à un représentant.

Ce conseil d'administration doit se réunir trois ou quatre fois par année. A leur assemblée annuelle, les membres procèdent à l'élection d'un comité exécutif qui se compose d'un président, d'un vice-président, d'un trésorier et d'un secrétaire. Ce comité exécutif est entièrement responsable de l'administration de la bibliothèque en autant qu'aucune des décisions prises ne va à l'encontre des politiques générales définies par le conseil d'administration. II n'y a aucun représentant du gouvernement ou de la Commission.
Le règlement général de chaque bibliothèque régionale est identique, à quelques détails près.

\section{Juridiction}

En principe, les bibliothèques centrales de prêt ont le mandat de desservir les municipalités de moins de 5,000 âmes. Cependant, dans des cas particuliers, des municipalités dont la population est comprise entre 10,000 et 15,000 âmes peuvent être admises, mais seulement après étude et avec autorisation expresse de la Commission des bibliothèques publiques. Chaque municipalité est libre d'adhérer ou non à la bibliothèque centrale de prêt.

Chaque municipalité désirant participer à la bibliothèque centrale de prêt devra d'abord se pourvoir, par voie de règlement municipal, d'une bibliothèque publique. Ceci fait, elle se liera par une convention avec la bibliothèque centrale de prêt, lui confiant un certain nombre de responsabilités et s'en imposant d'autres. La convention lie les parties pour deux ans et se renouvelle par tacite reconduction.

Dans l'ensemble, le partage est le suivant: la municipalité contractante nomme un représentant auprès de la corporation qu'est la bibliothèque centrale de prêt, paie sa contribution annuelle au taux établi, assume l'aménagement physique de sa bibliothèque (de fait une succursale) à moins d'avoir choisi le bibliobus, voit à l'engagement de son personnel. La bibliothèque centrale de prêt lui fournit les collections, l'infrastructure technique, les avis professionnels au personnel local, et, de manière générale les services attendus d'une bibliothèque régionale. La bibliothèque centrale de prêt n'a aucun pouvoir d'intervention directe dans les champs qui restent de responsabilité municipale.

Un autre type de rapport lie la bibliothèque centrale de prêt avec d'autres bibliothèques municipales de la région, celles qui sont autonomes, non soumises à la juridiction directe de la bibliothéque centrale de prêt. Elle devient alors une centrale technique où ces bi-

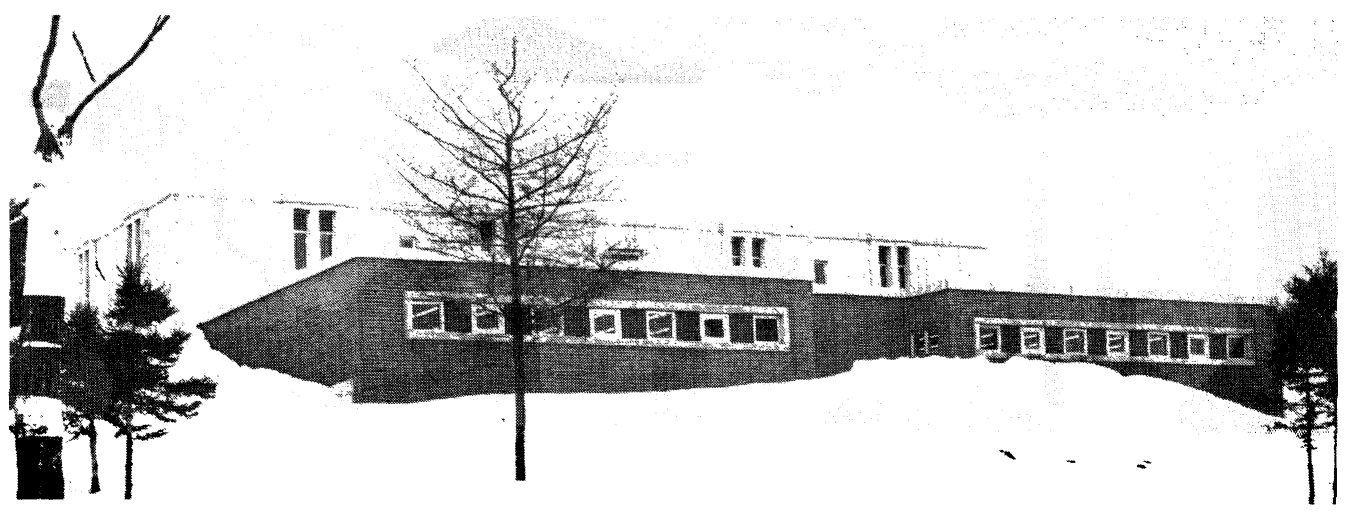

Siège social de la bibliothèque centrale de prêt du Saguenay-Lac-Saint-Jean. 
bliothèques centralisent le catalogage et la préparation matérielle de leurs collections. Elles ne sont pas représentées auprès de la bibliothèque centrale de prêt, mais collaborent avec elle sur ce point. Cette opération n'a pas été un choix spécifique voulu au départ; c'est plutôt l'importance relative de la bibliothèque centrale de prêt au niveau régional, et l'évident intérêt économique de chacun qui l'ont imposé.

\section{4-A. Juridiction}

En Colombie, il est intéressant de noter que l'ensemble des municipalités sises à l'intérieur des limites du territoire desservi par la bibliothèque régionale doivent intégrer les rangs de cette bibliothèque. Si une municipalité était subventionnée par la Commission avant la création de la bibliothèque régionale, cette subvention cesse aussitôt que la bibliothèque régionale entre en activité.

Les bibliothèques régionales peuvent, de leur côté, desservir toutes les municipalités indépendamment de leur population. C'est ainsi que la Bibliothèque régionale de Fraser Valley fournit le service à des villes de 60,000 et de 96,000 habitants. II y a donc une centralisation intégrale des services techniques pour toutes les bibliothèques publiques d'une région et une centralisation administrative complète susceptible d'entraîner des économies réelles.

La loi sur les bibliothèques publiques définit les pouvoirs et responsabilités de l'organisme, appelé bibliothèque régionale, et des municipalités ou districts scolaires contractants. C'est ainsi que la loi prévoit la formation du conseil d'administration par la nomination d'un représentant par chaque municipalité et district scolaire en plus de ses pouvoirs. Les représentants doivent se réunir au moins trois fois par année et ne peuvent sièger au conseil plus de deux années consécutives. Le conseil d'administration doit établir une bibliothèque publique satisfaisante, et pour cela, étabłir une bibliothèque centrale qui desservira la région par des succursales; il a le pouvoir de passer tout contrat aux fins précitées; il doit engager une personne qualifiée comme bibliothécaire qui sera à la fois secrétaire et administrateur en chef du conseil; il peut formuler des règlements pour la conduite des affaires ou pour l'utilisation des bibliothèques par le public; il peut accepter que telle succursale donne un service à un non résident et en fixer la cotisation; il doit tenir une bonne comptabilité et en soumettre la teneur à un comtable agréé; il doit contribuer aux bénéfices marginaux des employés; il doit préparer un rapport annuel; il a tout autre pouvoir nécessaire à la bonne marche de la bibliothèque.

Les éléments des règlements administratifs sont contenus en grande partie dans le texte de loi sur les bibliothèques publiques.

\section{Financement}

Les ressources financières des bibliothè- ques centrales de prêt du Québec proviennent essentiellement de trois sources: les municipalités contractantes $(10 \%)$, le Ministère des Affaires culturelles sur recommandation de la Commission des bibliothèques publiques $(80 \%)$ et les municipalités non-contractantes mais participant à la centralisation régionale des services techniques (10\%).

Le taux annuel de contribution des municipalités contractantes est déterminé par l'assemblée générale des membres. Actuellement, il est de $\$ 0.25$ per capita. La population résidente constitue donc le seul facteur déterminant la contribution et la somme totale disponible est aisément connue. Cependant, et en vertu des obligations qu'elles se sont imposées lors de la signature de la convention, les municipalités contractantes doivent rencontrer d'autres dépenses dont la somme égale sensiblement leur contribution annuelle mais n'apparaît pas au budget des bibliothèques centrales de prêt.

La Commission des bibliothèques publiques définit de son côté et pour chaque bibliothèque centrale de prêt, compte tenu de certains facteurs comme la population desservie, le taux de contribution des municipalités, l'importance démographique des unités municipales, une enveloppe de subvention qui pourrait être atteinte mais jamais dépassée.

Les municipalités non-membres mais dont les bibliothèques autonomes participent à la centralisation régionale des services techniques font connaître en temps opportun leurs besoins et budgets, de sorte que l'ampleur de cette opération soit connue d'avance.

Le tout constitue une enveloppe totale à l'intérieur de laquelle le budget devra nécessairement se loger. C'est la responsabilité de l'administrateur délégué et du conseil d'administration de l'élaborer, en fonction des programmes qu'ils ont choisis de réaliser. Avant que soit connu le montant exact de la subvention, dont l'importance est telle qu'il peut décider à lui seul de la survie des différents programmes, voire de la bibliothèque centrale de prêt elle-même, il aura fallu que l'administrateur délégué soutienne devant la Commission des Bibliothèques publiques les prévisions budgétaires soumises avec la demande de subvention et, du même coup, les programmes qui les sous-tendent.

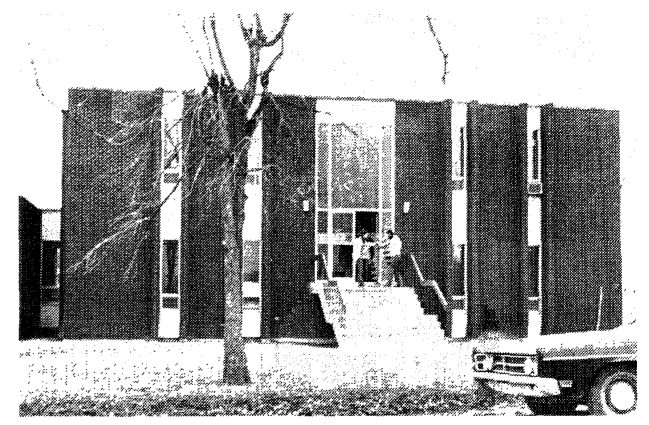

Siège social de la bibliothèque centrale de prêt de l'Outaouais. 


\begin{tabular}{|c|c|c|c|c|c|c|}
\hline \multirow[t]{2}{*}{ Poste budgétaire ${ }^{2}$} & \multicolumn{2}{|l|}{ B.C.P.M. } & \multicolumn{2}{|l|}{ B.C.P.O. } & \multicolumn{2}{|l|}{ B.C.P.L.S.J. } \\
\hline & $\$$ & $\%$ & $\$$ & $\%$ & $\$$ & $\%$ \\
\hline Salaires & 145,450 & 54.8 & 142,410 & 49.2 & 44,300 & 27.6 \\
\hline Volumes & 46,000 & 17.3 & 54,500 & 18.9 & 35,800 & 22.4 \\
\hline Films & 2,200 & 0.8 & 3,000 & 1.0 & - & - \\
\hline Disques & 7,500 & 2.8 & 6,000 & 2.0 & 3,000 & 1.9 \\
\hline Fournitures & 7,900 & 2.9 & 4,400 & 1.6 & 1,500 & 0.9 \\
\hline Communications & 9,900 & 3.8 & 10,100 & 3.6 & 8,300 & 5.1 \\
\hline Publicité & 6,500 & 2.4 & 4,850 & 1.7 & 2,560 & 1.6 \\
\hline Activités culturelles & 3,400 & 1.2 & 3,500 & 1.2 & 3,500 & 2.1 \\
\hline Service de la dette & 10,900 & 4.1 & 13,800 & 4.8 & 23,095 & 14.4 \\
\hline Immobilisations & 5,000 & 1.9 & 14,258 & 5.0 & 28,141 & 17.5 \\
\hline Automatisation & 3,000 & 1.1 & 6,000 & 2.0 & - & - \\
\hline $\begin{array}{l}\text { Matériel roulant } \\
\text { (entre. \& Rép.) }\end{array}$ & 8,000 & 2.0 & 7,000 & 2.4 & 1,000 & 0.6 \\
\hline $\begin{array}{c}\text { Achat d'oeuvres \& } \\
\text { encadrement }\end{array}$ & - & - & 1,500 & 0.5 & - & - \\
\hline Autres dépenses & 9,850 & 3.9 & 17,882 & 6.1 & 9,460 & 5.9 \\
\hline TOTAL & 265,600 & & 289,200 & & 160,656 & \\
\hline Population desservie & 111,933 & & 121,100 & & 54,000 & \\
\hline Per Capita & 2.37 & & 2.39 & & 2.97 & \\
\hline
\end{tabular}

\section{5-A. Financement}

Les bibliothèques régionales sont financées directement par les municipalités et les districts scolaires desservis, et par la Commission pour le développement des bibliothèques dans une faible proportion. Celle-ci fournit environ 9 pour cent du budget de chaque bibliothèque régionale.

Le budget des bibliothèques régionales est préparé par le bibliothécaire responsable et est approuvé par le conseil d'administration. Une fois les dépenses globales déterminées, ce montant est divisé par la somme totale de l'évaluation foncière de chaque municipalité et de chaque district scolaire. Ainsi les dépenses de 1972 de la bibliothèque régionale de l'lle de Vancouver se chiffrent à $\$ 647,762$. Si l'on soustrait de cette somme les revenus de la Commission et d'autres sources, les municipalités et les districts sco-

2. Pour le détail de ces postes, voir l'annexe. laires doivent débourser pour leur bibliothèque publique la somme de $\$ 641,602$. Or l'évaluation foncière de ces municipalités est de l'ordre de $\$ 572,859,176$. Le chiffre moyen établit est donc le 1.12 par mille. La municipalité de Duncan, par exemple, dont l'évaluation foncière est de $\$ 9,287,802$. devra payer $\$ 10$,402 à la bibliothèque régionale.

Une fois le montant fixé pour chaque municipalité, le représentant de la municipalité avise son conseil municipal de la dépense à effectuer pour la bibliothèque publique. Lorsque la facture est reçue par la municipalité, la loi prévoit que cette dernière doit acquitter cette facture ("shall be paid over by the Municipal Treasurer").

Dans le cas des districts scolaires, c'est-àdire pour les agglomérations de population nòn municipalisées incluses et desservies par la bibliothèque régionale, la facture est directement adressée au Ministre des finances de la province. Le directeur de la bibliothèque, à titre de secrétaire du conseil d'administration, écrit au Ministre des finan- 


\begin{tabular}{|c|c|c|c|c|c|c|}
\hline \multirow[t]{2}{*}{ Poste budgétaire } & \multicolumn{2}{|l|}{ B.R.I.V. } & \multicolumn{2}{|l|}{ B.R.F.V. } & \multicolumn{2}{|l|}{ B.R.O. } \\
\hline & $\$$ & $\%$ & $\$$ & $\%$ & $\$$ & $\%$ \\
\hline Salaires & 391,710 & 60.5 & 461,520 & 60.9 & $267,360^{1}$ & 60.6 \\
\hline Volumes & 151,500 & 23.5 & 216,000 & 28.3 & 120,000 & 27.3 \\
\hline Films & - & - & 10,000 & 1.3 & 5,050 & 1.2 \\
\hline Location & 20,302 & 3.0 & - & - & $1,080^{2}$ & 0.2 \\
\hline Ameublement & 30,000 & 5.0 & 8,500 & 1.1 & 10,068 & 2.2 \\
\hline Entretien & 5,000 & 0.7 & 6,400 & 0.7 & 3,900 & 0.9 \\
\hline Poste et transport & 2,500 & 0.4 & 2,200 & 0.3 & 2,000 & 0.4 \\
\hline Dépenses des véhicules & 10,000 & 1.4 & 7,000 & 0.8 & $7,500^{3}$ & 1.8 \\
\hline Capitalisation & 5,000 & 0.7 & 4,500 & 0.6 & 3,200 & 0.8 \\
\hline Nouveau véhicule & - & - & $7,000^{4}$ & 0.8 & 3,500 & 0.8 \\
\hline Assurance & 2,500 & 0.4 & 550 & 0.07 & 1,000 & 0.2 \\
\hline Comptable & 750 & 0.1 & - & - & 500 & 0.1 \\
\hline Frais bancaires & - & - & 2,700 & 0.33 & 450 & 0.1 \\
\hline $\begin{array}{l}\text { Frais de réunion } \\
\text { (conseil, bibliothé- } \\
\text { caires) }\end{array}$ & 5,000 & 0.7 & 4,000 & 0.5 & 5,300 & 1.3 \\
\hline Promotion \& publicité & 4,000 & 0.6 & - & - & - & - \\
\hline Enquête & - & - & 15,000 & 2.0 & - & - \\
\hline Papeterie \& impression & - & - & 16,000 & 2.0 & - & - \\
\hline $\begin{array}{l}\text { Utilités } \\
\text { (tél., télex, etc.) }\end{array}$ & 19,500 & 3.0 & - & - & 1,800 & 0.4 \\
\hline Dépenses diverses & - & - & 2,490 & 0.3 & 2,320 & 0.6 \\
\hline Catalogue (projet IBM) & - & - & - & - & $4,500^{5}$ & 1.1 \\
\hline 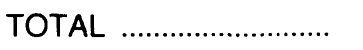 & $\$ 647,762$ & & $\$ 756,860$ & & $\$ 441,578$ & \\
\hline Population desservie.... & 145,000 & & 372,301 & & 98,228 & \\
\hline Financement .................... & $\begin{array}{l}1.12 \mathrm{de} \\
\$ 572,859,17\end{array}$ & & $\begin{array}{l}50 \%: 40 \text { de } \\
\$ 809,324,170 \\
50 \%: \$ 0.88 \times \\
372,301 \text { (h.) }\end{array}$ & & $\begin{array}{l}1.2 \mathrm{de} \\
\$ 331,966,10\end{array}$ & \\
\hline Per capita & 4.47 & & 2.03 & & 4.50 & \\
\hline $\begin{array}{l}\text { (1) Les Bibliotheques régionales } \\
\text { et les commis responsables des } \\
\text { cas de la Bibliotheque régionale } \\
\text { quelques autres. } \\
\text { (2) La Bibliotheque régionale de } \\
\text { le coût de location des succursa } \\
\text { faire. Dans le cas d'Okanagan, c } \\
\text { sée en grande partie par les mu }\end{array}$ & $\begin{array}{l}\text { aient les bibliothéc } \\
\text { lccursales, sauf da } \\
\text { de Fraser Valley } \\
\text { le de Vancouver de } \\
\text { s. Elle est la seule } \\
\text { te somme est remb } \\
\text { cipalités. }\end{array}$ & & $\begin{array}{l}\text { (3) La Bibliothè } \\
\text { sede trois bibliob } \\
\text { La Bibliotheque } \\
\text { bibliobus; celle d } \\
\text { nette. } \\
\text { (4) Ce chiffre est } \\
\text { le bibliobus. Le } \\
\$ 11,000 \text {. } \\
\text { (5) La mise sur or } \\
\text { mes a coûté } \$ 9,\end{array}$ & $\begin{array}{l}\text { Ue régi } \\
\text { us, deu } \\
\text { úgional } \\
\text { 'Okanag } \\
\text { tle prix } \\
\text { coût to } \\
\text { rinateu } \\
\text { 536. en }\end{array}$ & $\begin{array}{l}\text { nale de l'lle de } V \\
\text { r camionnettes et } \\
\text { de Fraser Valley } \\
\text { an, deux bibliobus } \\
\text { du châssis utilisé p } \\
\text { al s'élève approx } \\
\text { de la collection de } \\
1970 \text { et } \$ 4,789 \text {. e }\end{array}$ & $\begin{array}{l}\text { ancouver pos- } \\
\text { 4 automobiles. } \\
\text { possede deux } \\
\text { et une camion- } \\
\text { pour construire } \\
\text { imativement à } \\
140,000 \text { volu- } \\
1971 \text {. }\end{array}$ \\
\hline
\end{tabular}


ces dans les termes suivants: "Je, monsieur $x$, secrétaire du conseil d'administration, certifie que, en conformité avec la loi des bibliothèques publiques, le conseil d'administration a voté, pour fins de bibliothèques, les sommes suivantes qui ont été établies à partir de l'évaluation foncière des districts scolaires inclus dans le territoire de la bibliothèque régionale et pour les territoires ruraux inclus dans les districts scolaires suivants..." En 1971, la bibliothèque régionale de l'lle de Vancouver adressait une facture de $\$ 345,522$ au Ministre des Finances.

\section{Moyens d'action \\ 1) Livres}

Le trois bibliothèques centrales de prêt du Québec procèdent sensiblement de la même façon pour la circulation de la collection de livres, utilisant soit le prêt direct par le bibliobus, soit le prêt indirect par les bibliothèques municipales affiliées dites succursales.

La Bibliothèque centrale de prêt de la Mauricie et la Bibliothèque centrale de prêt de l'Outaouais utilisent chacune un bibliobus. II s'agit de véhicules considérables pouvant contenir 3,000 volumes, des films, des disques et l'êquipement requis pour l'exposition de tableaux, photographies ou gravures. Leur valeur actuelle de remplacement se situe aux environs de $\$ 45,000$. En principe, ils sont utilisés pour desservir les municipalités de moins de 1,000 habitants qu'ils visiteront une fois par mois chacune pour un prêt de durée variable.

Les succursales reçoivent leurs volumes de la bibliothèque centrale de prêt qui les échange tous les quatre mois dans des proportions différentes selon les bibliothèques centrales de prêt. Elles disposent, sauf en Mauricie, du catalogue de leur collection mais non pas du catalogue général. Leurs usagers utilisent beaucoup le service de demandes spéciales (annuellement 7,500 en Mauricie et 1,500 dans l'Outaouais) pour lesquelles ils reçoivent satisfaction dans $75 \%$ des cas, par la poste. En plus des livres, elles disposent de périodiques, qui leur sont fournis par abonnements directs dont la valeur varie selon la population que chacune dessert. Le personnel en charge des succursales est choisi localement, soit par l'administration municipale elle-même dont c'est une des responsabilités selon la convention, ou par délégation: le plus souvent, il s'agit de services bénévoles, avec les problèmes et avantages que cela comporte.

\section{2) Films}

Deux bibliothèques centrales de prêt, celles de la Mauricie et de l'Outaouais, possèdent des collections de films, 800 et 1,176 respectivement. Ces collections comprennent en majorité du court métrage documentaire provenant, par dépôt ou achat, de l'Office National du Film. Toutefois, les achats récents comprennent beaucoup de longs et courts métrages de création, en $16 \mathrm{~mm}$. et 8 $\mathrm{mm}$., choisis parmi les grands titres du cinéma américain d'avant 1930. Ces films sont disponibles gratuitement, sauf le coût du transport dans un sens, à tous les abonnés et à tous les groupements du territoire de chaque bibliothèque centrale de prêt. Le service s'effectue à partir du siège social.

\section{3) Disques}

Les trois bibliothèques centrales de prêt ont des collections de disques qui sont décentralisées dans les succursales comme les livres et selon les mêmes modalités. Le service de demandes spéciales n'existe cependant pas pour eux, essentiellement parce que les collections sont de taille insuffisante pour l'alimenter.

\section{4) Oeuvres d'art}

Les bibliothèques centrales de prêt de l'Outaouais et du Saguenay-Lac-Saint-Jean ont des collections d'oeuvres d'art qui sont mises à la disposition des individus sous forme de prêt individuel ou des succursales pour activités de groupe et prêt à leurs usagers.

\section{5) Manifestations culturelles diverses}

Dans la majorité des municipalités desservies, les bibliothèques centrales de prêt sont, de fait, les seuls pourvoyeurs de biens culturels en dehors des institutions d'enseignement. Cette situation, indépendamment des options de principes qui auraient pu être prises antérieurement, leur a imposé la nécessité d'élargir la notion traditionnelle de bibliothèque en conformité avec la tendance suivie par l'ensemble des bibliothèques américaines et européennes. Car, pour toutes sortes de besoins culturels non imprimés, les usagers se sont retournés vers elles, spontanément. Et inversement, le caractère vivant de ces manifestations a toujours été un attrait particulier pour des gens qui n'utilisaient pas la bibliothèque avant d'en avoir la révélation en d'autres occasions. Ces deux motifs ont donc incité les trois bibliothèques à tenir systématiquement des manifestations culturelles diverses de tous caractères manifestations qui sont devenues un élément essentiel de leur action.

\section{6-A. Moyens d'action \\ 1) Livres}

Les trois bibliothèques régionales de la Colombie procèdent de la même façon pour la circulation de la collection de livres. II y a le prêt direct par bibliobus et le prêt par l'intermédiaire des succursales.

Bibliobus: les bibliobus de ces bibliothèques diffèrent des nôtres en ce qu'ils sont plus petits et qu'ils coûtent, par le fait même, moins chers, soit $\$ 11,000$ chacun en moyenne. Ils contiennent environ 2,000 volumes; le nombre d'arrêts varie de 100 à 240 et chaque arrêt dure en moyenne 20 minutes.

Succursales: les succursales sont dirigées très souvent par du personnel professionnel rémunéré par la Bibliothèque régionale. Cette situation explique, à notre avis, l'efficacité et le niveau du prêt des volumes. On connâit l'importance des demandes spéciales. Ces dernières sont très élevées dans les bibliothèques régionales de la Colombie Britannique. Elles varient de 19,793 à 83,796. Les succursales possèdent un catalogue imprimé de tous les volumes de la Bibliothèque régionale. Celle de l'lle de Vancouver dispose d'un télex 
pour chaque sous-région qui les relie au siège social. Les volumes sont livrés par camionnette chaque semaine à la succursale demanderesse. Les collections de volumes sont variées et la collection de la Commission pour le développement des bibliothèques publiques sert à chaque bibliothèque régionale. Une partie de la collection des succursales est échangée régulièrement, de telle sorte que les abonnés ont toujours des nouveautés.

\section{2) Films}

Deux bibliothèques régionales distribuent des films; l'une possède une collection de 761 films et l'autre de 1,031 films. Le prêt des films s'effectue à partir du siège social pour la plupart des demandes et par l'intermédiaire de certaines succursales où il y a des blocs de films. Les films sont loués et le prix de location varie de $\$ 0.50$ à $\$ 1.25$.

\section{3) Oeuvres d'art}

Il est assez surprenant de constater que la bibliothèque régionale de Vancouver n'offre pas de films à ses abonnés, mais, par contre, dispose d'une collection de 500 oeuvres d'art. Ces oeuvres d'art, mises à la disposition des succursales, sont prêtées aux individus. Le prêt des oeuvres d'art intéresse beaucoup d'abonnés.

\section{Méthodes de contrôle des collections}

Le contrôle externe est effectué par la compilation des statistiques de prêt que les succursales sont tenues de compiler et de communiquer au siège social de leur bibliothèque centrale de prêt sur des formules préparées à cette fin; ceci vaut pour toutes leurs collections, livres, périodiques, disques ou oeuvres d'art. De plus, les responsables de succursales reçoivent régulièrement la visite d'agents culturels de la bibliothèque centrale de prêt qui sont polyvalents et agissent comme agents de liaison et conseillers techniques auprès des responsables.

Le contrôle interne s'effectue à l'aide de deux fichiers. Le premier est dit "quadrillé" parce que la fiche de chaque titre porte au verso un quadrillage de localisation. Chaque titre, en effet, est représenté par une fiche auteur qui mentionne le nombre total d'exemplaires: le dessin du quadrillage est tel qu'il est possible d'enregistrer les mouvements d'un volume entre le siège social et les diverses succursales sans ambiguïté. Ce fichier permet de connaître à tout moment la localisation de chaque ouvrage à partir de la notice bibliographique.

Le second est dit "de succursales". Chaque volume contient deux fiches de prêt: l'une accompagne le volume dans la succursale, l'autre demeure à la centrale. Les fiches conservées sont classées par succursales et sous-classées topographiquement. Ce second fichier permet donc de connaître l'inventaire de chaque collection de succursale et de vérifier la conformité de sa composition avec le dessin général de collection qui aura été arrêté pour répondre aux besoins particuliers de telle municipalité.

\section{7-A. Méthodes de contrôle}

Le contrôle externe: chaque succursale adresse au siège social ses statistiques mensuelles sur des feuilles préparées à cette fin. Le personnel des succursales étant rémunéré par la bibliothèque régionale, il est facile de concevoir que les statistiques sont adressées régulièrement au siège social. Chaque volume a sa carte du livre qui est conservée à la succursale jusqu'à son retour.

Le contrôle interne: chaque bibliothèque dispose d'un fichier collectif de localisation qui permet de retracer n'importe quel volume assez rapidement. Plusieurs opérations sont requises lors des rotations ou de l'envoi de nouveaux volumes dans les succursales. II y a tout d'abord une fiche contenant les différents exemplaires du même titre, et on inscrit sous le numéro de tel exemplaire, la date de l'expédition et le numéro de la succursale où le volume est adressé. De plus, chaque volume possède deux cartes du livre dont l'une est conservée au siège social et sur laquelle on inscrit la date d'expédition et le numéro de la succursale où le volume est expédié.

\section{Automatisation}

Au Québec, les trois bibliothèques centrales de prêt mènent actuellement une étude conjointe sur l'opportunité et la possibilité d'utiliser l'informatique pour répondre à certains objectifs. II s'agit essentiellement d'obtenir une gestion automatique des collections (qui permettrait la disparition des deux fichiers actuels de contrôle dont la mise à jour constante est très coûteuse) et l'impression d'un catalogue général pour chaque bibliothèque centrale de prêt, puis d'un catalogue local pour chacune de leurs succursales; chacun de ces catalogues comprendrait quatre parties: enfants, adultes alphabétique-auteurs, adultes alphabétique-titres et adultes-sujets. D'autres applications sont également étudiées: préparation de bibliographies, de listes de nouveautés, d'un catalogue collectif, etc...

Dans son état actuel, l'étude démontre qu'il n'y a pas d'économie en perspective, si ce n'est la possibilité d'une amélioration considérable des services pour un coût additionnel moindre. Elle révèle également qu'il n'y a aucun intérêt économique, bien au contraire, à utiliser l'informatique à la seule fin d'imprimer un catalogue général, mais qu'il importe de répartir les coûts de base sur un nombre relativement élevé d'appications. C'est à suivre.

\section{8-A. Automatisation}

En Colombie, deux bibliothèques régionales ont mis leur collection sur ordinateur et achètent du temps à la compagnie I.B.M. La troisième bibliothèque effectue cette opération manuellement. Dans les trois cas, les abonnés ont à leur disposition un catalogue imprimé de la collection de livres. La publica- 
tion d'un catalogue réalisé manuellement occupe une secrétaire pendant quatre mois.

C'est dans le but d'obtenir un rendement maximum d'utilisation de la collection que les bibliothécaires en sont arrivés à la conclusion qu'il était essentiel d'avoir un catalogue imprimé de la collection, divisé en trois parties: auteur, titre et sujet. L'analyste engagé par la bibliothèque régionale concluait que les avantages de l'utilisation d'un ordinateur sont les suivants:

- présence d'un catalogue imprimé de la collection dans toutes les succursales;

- la mise à jour de la collection coûte peu;

- diminution du coût du processus techni-

que;

- possibilité de participer à un catalogue collectif.

Le coût envisagé par les administrateurs de la bibliothèque d'Okanagan, au moment de l'étude, s'élèverait à $\$ 10,000$ pour la première année à cause de la mise de la collection sur cartes perforées. Ils envisageaient une dépense de $\$ 3,500$ pour les années subséquentes.

Le coût de la mise sur ordinateur de la bibliothèque de l'lle de Vancouver s'est élevé à $\$ 15,500$ pour la première année et à $\$ 6,200$ pour les années subséquentes. II s'agissait d'une collection de 72,000 titres.

Ni l'une ni l'autre n'a encore envisagé l'automation du système du prêt des volumes effectué à partir de la bibliothèque centrale dans les succursales, pas plus d'ailleurs que automation des données statistiques et de la comptabilité.

\section{Communications}

Les trois bibliothèques centrales de prêt attachent une grande importance aux communications entre le siège social et les succursales. Des agents culturels, chargés personnellement de représenter la bibliothèque dans la multitude de ses service, font la navette pour aider les responsables, recevoir leurs doléances et suggestions, conseiller les usagers du bibliobus et des succursales, très souvent être seulement présents. De même, les responsables sont régulièrement réunis au siège social ou ailleurs, toujours aux frais de la bibliothèque centrale de prêt pour des motifs d'ordre technique et social. Nous connaissons des sauteries qui ont réglé pas mal plus de problèmes que certains ateliers de travail. Ces initiatives ont permis de créer entre les différentes personnes participant au projet un esprit de corps remarquable; de multiples exemples pourraient être donnés d'améliorations notables qui sont venues de suggestions des "non-instruits".

Mais, sont aussi fondamentales les communications des bibliothèques centrales de prêt avec leurs milieux que celles des succursales avec leurs micro-milieux. Ainsi en est-il d'ententes entre Communication-Québec et des bibliothèques centrales de prêt qui ont permis à des succursales de devenir des centres d'information extrêmement vivants. Des échanges de renseignements entre les bibliothèques centrales de prêt et les services d'éducation permanente de Commissions scolaires régionales ont également permis l'établissement de services bibliographiques très actualisés et vivifiè de façon sensible certaines succursales. II en est de même de certains échanges de nature semblable entre les bibliothèques centrales de prêt et des associations fortement présentes dans le milieu rural, telle l'Association Féminine d'Education et d'Action Sociale; on pourrait encore citer les Conseils régionaux de Loisirs, les Bureaux d'aménagement culturels et bien d'autres. En outre, les bibliothèques centrales collaborent sans cesse à l'impression de dépliants publicitaires, d'affiches, etc... au bénéfice des succursales.

Ces rapports sont essentiels parce qu'ils informent les bibliothèques centrales de prêt des besoins du milieu et leur assurent ainsi une meilleure intégration. Mais ils sont bien réalisés dans la mesure où les succursales elles-mêmes y participent. Les services bibliographiques seront utiles pourvu que les volumes et documents recommandés soient disponibles dans la succursale fréquentée par le requérant et dans le cas où les besoins particuliers qu'ils sous-tendent auront été considérés au moment d'effectuer la rotation de la collection; sans la réalisation de ces conditions, la machine tourne bien, mais à vide.

La réussite ou l'échec de ces tentatives est déterminante si les bibliothèques centrales de prêt veulent échapper au danger de se voir apposer une étiquette purement récréative. Déjà, en 1966, l'enquête réalisée par Gilbert Gagnon faisait ressortir sans pouvoir y répondre une discontinuité profonde entre la motivation à lire des usagers et l'image qu'ils se font de "la" bibliothèque publique. "La" bibliothèque publique, peut-être était-ce "la leur". Le défi est là.

\section{9-A. Communications}

Les trois bibliothèques régionales apportent une attention particulière aux communications, c'est-à-dire aux relations entre les succursales et le siège social. On se souvient que les succursales importantes de la Bibliothèque régionale de l'lle de Vancouver sont reliées entre elles par des télex, et que le bibliothécaire attaché à ces succursales dispose d'une automobile pour visiter les succursales moins importantes de sa sous-région.

Les bibliothèques régionales utilisent des automobiles et des camionnettes pour transporter les volumes réclamés par telle ou telle succursale, et des bibliobus visitent les agglomérations desservies, à un rythme de deux à trois semaines.

De plus, le personnel professionnel rémunéré par la bibliothèque régionale se réunit mensuellement au siège social afin de discuter des problèmes de chaque cellule du système. 


\section{Conclusion}

Cette initiative de la Commission des Bibliothèques publiques du Ministère des Affaires culturelles du Québec (création de bibliothèque régionales) a permis d'amorcer ce que l'on appelle communément la "démocratisation de l'art et de la culture". Ces termes ne sont pas de vains mots lorsque des citoyens de régions éloignées, dépourvus financièrement, isolés par l'absence de moyens de communication adéquats, ont accès gratuitement aux biens culturels. II y a encore beaucoup de régions à desservir, beaucoup d'énergies à regrouper et de nombreux préjugés à vaincre.

\section{Liste des documents consultés (Québec)}

"La Bibliothèque centrale de prêt de l'Outaouais; accessibilité aux biens culturels", Le Pachyderme, no spécial (août 1972).

Bibliothèque centrale de prêt de la Mauricie. Budget d'opération 1972-1973. Trois-rivières, s.d.

Rapport annuel, 1971-1972, par Jacques Panneton. Trois-Rivières, 1972.

res, s.d.

Règlement général. Trois-Riviè-

Bibliothèque centrale de prêt du SaguenayLac-Saint-Jean. Budget d'opération, 1972-1973. Alma, s.d.

Rapport annuel, 1971-1972, par Philippe Sauvageau. Alma, 1972.

Règlement général. Alma, s.d.

Bibliothèque centrale de prêt de l'Outaouais. Budget d'opération, 1972-1973. Hull, s.d.

Rapport annuel, 1971-1972, par Philippe Sauvageau. Hull, 1972.

Règlement général. Hull, s.d.

Québec (province). Ministère des affaires culturelles. Service des bibliothèques publiques. Découpage du territoire québécois en régions de bibliothèques, par Gilbert Gagnon. Québec, 1967.

La direction des bibliothèques publiques du Québec, par Gérard Martin. Québec, 1970.

Habitudes et comportements des lecteurs, région de la Mauricie, par Gilbert Gagnon. Québec, 1970.

Bibliothèques publiques du Québec; rapport statistique, 1971. Québec, 1972.
Liste des documents consultés (Colombie Britannique)

British Columbia. The Library Development Commission. Public Libraries Statistics, 1970. Victoria, 1971.

1971.

Annual Report, 1970. Victoria,

Centennial Citizens' Conference on Libraries. Libraries: vital to tomorrow's world. Vancouver, 1971.

Committee on Library Development. A Proposal for Province-Wide Organization of Library Services in British Columbia, submitted to the British Columbia Library Development Commission. B.C., 1971.

Fraser Valley Regional Library. 41st Annual Report 1971, by Ronald Ley. Abbotsford, 1972.

Film 71-72. Abbotsford, s.d.

Ley, Ronald. A Brief History of the Fraser Valley Regional Library and an Outline of the Library System Together with a List of Officers and Members of the Board of Management 1934-1971. Abbotsford, Fraser Valley Regional Library, 1971.

Okanagan Regional Library. Annual Report 1971, by W.P. Lofts. Kelowna, 1972.

\section{Oriented Library Catalogue; prepared by Georges Veenhuysen and Geoff Heak. Kelowna, 1969.}

Vancouver Island Regional Library. Annual Report for the Year Ended December 31st, 1971, by Fred T. White. Nanaimo, 1972.

The Key; an unverified history of the Vancouver Island Regional Library in celebration of Canada's Centennial by the members of the branch libraries. Nanaimo, edited by Betty Rogers, 1967.

White, Fred T. "No problem an I.B.M. Venture" , British Columbia Library Quarterly, Vol. 30, no. 4 (April 1967), 9-11.

ANNEXE

Nomenclature détaillée des postes budgétaires

Salaires:

Charges sociales incluses

Volumes:

Livres

Périodiques

Reliure et réparation

Films:

Diapositives incluses 
Disques:

Evident

Fournitures:

Papeterie, fiches, etc...

Articles de bureau

Matériel pour la préparation matérielle

Communications:

Frais de voyage

Téléphone et télégraphe

Poste

Port

Publicité:

Bulletin

Affiches, dépliants, etc...

Annonces payées

Réceptions et conférences

Frais de représentation

Activités culturelles:

Expositions

Projections de films

Concerts

Théâtre

Service de la dette:

Remboursement d'emprunts

Immobilisations:

Terrain

Bâtiments

Mobilier et équipement

Projecteurs

Matériel roulant

Autres dépenses:

Frais bancaires

Frais légaux

Loyer:

Bâtiments

Equipement

Assurances

Chauffage

Eclairage

Taxes

Réparation et entretien:

Terrain

Bâtiments

Mobilier et équipement

Matériel roulant

Dépenses diverses

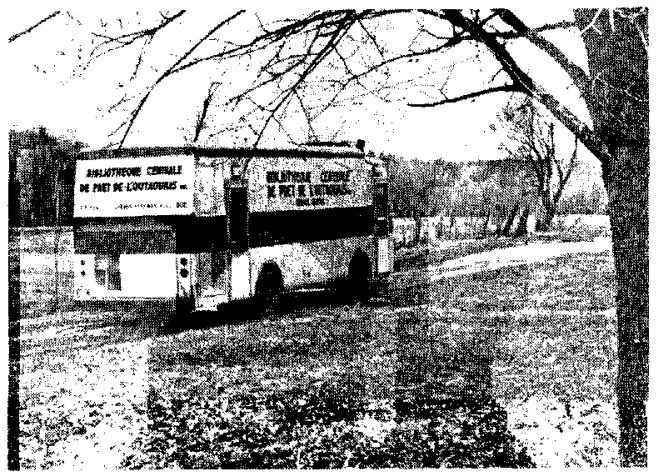

DU LANGAGE

A. Martinet et M. Merleau-Ponty par GHYSLAIN CHARRON

[SBN-0-7766-101] -2

$15,5 \times 23,5 \mathrm{~cm} ., 200$ pages. - Prix: $\$ 4.50$.

ANTOINE DU PERIER

LES AMOURS DE PISTION

ET DE FORTUNIE

Texte critique avec Introduction et Notes par ROMÉO ARBOUR

ISBN-0-7766-4152-2

$15 \times 22 \mathrm{~cm}$., 152 pages.-Prix: $\$ 3.75$

En vente chez votre libraire et aux

ÉDITIONS DE L'UNIVERSITÉ

D'OTTAWA

Ottawa, Ontario, Canada, K1N 6N5 